\title{
Estudo sobre abandono e não adesão ao tratamento em Centros de Atenção Psicossocial
}

\author{
Study on patient dropout and non-adherence to mental health \\ treatment in Psychosocial Care Centers
}

Noemi Bandeira', Carlos Alberto dos Santos Treichel' ${ }^{\mathbf{1}}$, Rosana Teresa Onocko-Campos ${ }^{\mathbf{1}}$

DOI: $10.1590 / 0103-11042020 E 310$

RESUMO O objetivo deste estudo foi investigar os motivos pelos quais os usuários que estavam cadastrados nos Centros de Atenção Psicossocial III de Campinas (SP), durante a realização de um censo de serviços, não foram localizados, bem como identificar e caracterizar aqueles que haviam abandonado ou não aderido ao tratamento. Trata-se de um estudo transversal, analítico, de base documental que utilizou os diários de campo da pesquisa e o prontuário dos usuários nos serviços. Entre os usuários que não foram localizados, $17,8 \%(\mathrm{n}=70)$ haviam abandonado ou não aderido ao tratamento. Esse número era composto, majoritariamente, por homens, com média de idade de 39,9 anos (DP=13,7), cujo diagnóstico mais frequente era o de esquizofrenia. Quanto ao tempo de permanência, verificou-se que os usuários haviam passado mais de um ano ou menos de um mês nos serviços. Destaca-se que, inicialmente, 40,3\% dos casos haviam sido reportados como altas, enquanto, no final do estudo, observou-se que apenas $2,5 \%$ abrangiam verdadeiras altas clínica. Por meio deste estudo, foi possível identificar que, além da falta de dados nos prontuários, a prática da alta administrativa dificulta a identificação dos casos de abandono ou não adesão ao tratamento, prejudicando a sistematização dos dados acerca da continuidade do cuidado.

PALAVRAS-CHAVE Pacientes desistentes do tratamento. Saúde mental. Serviços comunitários de saúde mental.

ABSTRACT The aim of this study was to investigate the reasons why users who were registered in Psychosocial Care Centers type III of Campinas (SP) during a census of services were not located, as well as to identify and characterize those who had abandoned or not adhered to treatment. This is a cross-sectional, analytical, documentary-based study that used field diaries and users' medical records. Among users who had not been located, $17.8 \%(n=70)$ had abandoned or not adhered to treatment. Most of these were men, with a mean age of 39.9 years ( $S D=13.7$ ), whose most frequent diagnosis was schizophrenia. Regarding length of attending; it was found that users had attended either more than 1 year or less than 1 month in the services. It is noteworthy that, initially, $40.3 \%$ of the investigated cases had been reported as discharges while at the end of the study it was observed that only $2.5 \%$ of the cases covered true clinical discharges. Thus, it was identified that, besides the lack of data in the medical records, the practice of administrative discharge makes it difficult to identify cases of abandonment or non-adherence to treatment, thus impairing the systematization of data about continuity of care. 


\section{Introdução}

A partir das conquistas do Movimento Antimanicomial, que deu origem à Reforma Psiquiátrica Brasileira, a política de saúde mental e a organização dos serviços de cuidado às pessoas com transtornos mentais no Brasil modificaram-se radicalmente nos últimos anos. Em 2001, o Congresso Nacional Brasileiro aprovou a Lei ${ }^{\circ} 10.216$, que atribui ao Estado a responsabilidade de consolidar a mudança do sistema assistencial e garantir aos usuários da saúde mental um cuidado não excludente, humanitário e resolutivo'.

Em 2002, a Portaria n ${ }^{\circ} 336$ do Ministério da Saúde estabeleceu as diretrizes de funcionamento dos Centros de Atenção Psicossocial (Caps), que devem privilegiar a criação de projetos terapêuticos singulares e a reabilitação psicossocial dos usuários, em meio aberto, de forma menos invasiva, priorizando o vínculo com a família e a comunidade, a liberdade e a garantia dos direitos humano ${ }^{2}$.

A partir do crescimento do número de Caps nas últimas décadas, culminando, em 2014, no número de 2.209 unidades instaladas ${ }^{3}$, houve também o aumento do número de estudos conduzidos com populações oriundas desses serviços. No campo dos estudos quantitativos, destacam-se aqueles de perfil ${ }^{4}$ de satisfação com os serviços ${ }^{5}$, mudanças percebidas ${ }^{6}$, entre outros. No entanto, ressalta-se que, apesar de fornecerem informações valiosas para organização e avaliação desses serviços, os estudos conduzidos até o momento são majoritariamente realizados com amostras intencionais, havendo poucos estudos com amostragem probabilística ou censos.

Parte disso pode-se atribuir ao fato de as pesquisas conduzidas com usuários de Caps esbarrarem em uma série de questões logísticas que dificultam o trabalho de campo ${ }^{7}$, entre as quais, destacam-se: a baixa qualidade dos dados disponibilizados pelos serviços (desorganização, falta ou desatualização dos registros); e, ainda, problemas relacionados com a falta de continuidade do tratamento dos usuários ${ }^{8}$.
Diante dessa realidade, além da opção pelo trabalho com amostras intencionais, é comum que haja um elevado percentual de perdas nos estudos conduzidos com essas populações. Enquanto são encontrados estudos conduzidos de forma intencional com usuários de Caps que relatam perdas de $0,19 \% 6$ e 6,6\% ${ }^{9}$, estudos conduzidos com amostras aleatórias de familiares de usuários reportaram perdas de $22,3 \%{ }^{10}$ e $52,4 \%{ }^{11}$. Contudo, não há aprofundamento acerca dos motivos e/ ou características dessas perdas.

Na pesquisa 'Inquérito sobre o acesso a partir da Atenção Básica, o funcionamento e a utilização da Atenção Especializada para quatro condições traçadoras em quatro grandes cidades brasileiras' (AcesSUS), que buscou incluir todos os usuários que foram referenciados ou chegaram por demanda espontânea aos Caps de Campinas (SP), as perdas relacionadas com a não localização dos sujeitos corresponderam a 41,8\% $(n=394)$ dos potenciais elegíveis.

Levando em conta que se tratava de usuários que, segundo os dados disponíveis no momento da pesquisa, deveriam estar frequentando os serviços, foram suscitadas dúvidas quanto aos motivos que levaram a não localização desses usuários. Entre as hipóteses levantadas, figurava a possibilidade de abandono ou não aderência ao tratamento, aspectos relacionados com o fenômeno da descontinuidade dos tratamentos ${ }^{12}$, que, em nível global, constitui um dos principais desafios no cuidado às pessoas com transtornos mentais ${ }^{13}$.

Destaca-se que em um levantamento realizado pela Organização Mundial da Saúde em 24 países $^{\mathbf{1 4}}$, de forma global, a evasão de serviços de saúde mental foi de $31,7 \%$. De forma estratificada, essa evasão correspondeu a $26,3 \%$ em países de alta renda, $45,1 \%$ em países de renda média-alta e 37,6\% em países de renda média/baixa.

Apesar da magnitude do problema, especialmente no cenário nacional, são encontrados poucos estudos documentados sobre o tema. 
Até o momento, por meio de uma revisão conduzida na base de dados Scientific Electronic Library Online (SciELO) no mês de julho de 2019, foi possível rastrear três estudos que abordaram diretamente o abandono do tratamento em serviços de saúde mental15-17.

Nesse sentido, este estudo teve como objetivo investigar os motivos pelos quais os usuários que estavam cadastrados nos Caps III de Campinas (SP), durante a realização da pesquisa AcesSUS, não foram localizados, identificando e caracterizando aqueles que haviam abandonado ou não aderido ao tratamento.

\section{Metodologia}

Trata-se de um estudo transversal, analítico, de base documental, conduzido a partir do estudo de prontuários de usuários de seis Caps do tipo III no município de Campinas (SP).

Ele é recorte da pesquisa AcesSUS, que, entre outras questões, analisou o acesso aos serviços a partir da atenção básica, além do funcionamento e da utilização da atenção especializada para pessoas com transtorno grave de saúde mental.

No censo de serviços supracitado, o intuito foi incluir na pesquisa todos os usuários que haviam sido referenciados ou chegaram por demanda espontânea aos Caps nos três anos que antecederam a coleta. Contudo, 41,8\% (n=394) desses usuários não foram localizados.

Dessa forma, o presente estudo objetivou investigar os motivos que impossibilitaram a localização desses usuários bem como identificar e caracterizar os usuários que, entre aqueles, abandonaram ou não aderiram ao tratamento. Para isso, foram utilizados como material de investigação os diários de campo dos coletadores e os prontuários dos usuários nos serviços - tanto os que estavam ativos como aqueles que constavam no arquivo morto. Nos diários de campo, haviam sido registradas as seguintes informações: nome completo; número de telefone; o Caps e a equipe em que o usuário estava cadastrado; a forma e a quantidade de tentativas de contato com este; os motivos alegados pelos familiares ou pelas equipes para a não localização do usuário; e relatos de observações orais feitas pelos profissionais sobre a condição do usuário.

Após a organização desses dados do diário de campo, iniciou-se a investigação nos prontuários, ambas conduzidas pela pesquisadora principal deste estudo que utilizou uma tabela própria para compilação de dados acerca da história clínica dos usuários, assim como das suas características sociodemográficas. Inicialmente, os dados foram digitados no software Microsoft Excel ${ }^{\circledR} \mathrm{e}$, posteriormente, convertidos para o pacote estatístico Stata 11 (Stata Corp., College Station, Estados Unidos), em que foram conduzidas as análises.

As variáveis estudadas foram sexo (masculino; feminino), idade (18 a 29 anos; 30 a 39 anos; 40 a 49 anos; 50 a 59 anos; 60 anos ou mais), estado civil (solteiro(a); casado(a); divorciado(a)), cor (preta; parda; branca), diagnósticos (esquizofrenia; transtorno afetivo bipolar; depressão; esquizofrenia concomitante ao uso abusivo de substâncias; outros), tempo no Caps (até 30 dias; até 3 meses; até 6 meses; até 1 ano; mais de 1 ano).

Para análise dos dados, foi utilizada estatística descritiva por meio da qual foram calculadas as médias para variáveis numéricas bem como seus respectivos desvios-padrão, além de cálculo de prevalência acerca de cada um dos estratos estudados. Os cálculos foram realizados com base nos dados válidos; os dados faltantes (missings) foram excluídos da análise.

$\mathrm{O}$ estudo foi submetido e aprovado pelo Comitê de Ética em Pesquisa da Faculdade de Ciências Médicas da Universidade Estadual de Campinas por meio do parecer técnico $\mathrm{n}^{\circ}$ 2.225.109. Sua realização se deu atendendo as normas e diretrizes brasileiras de regulamentação de pesquisas envolvendo seres humanos

- Resolução CNS no 466/2012 e garantindo-se o anonimato dos sujeitos. 


\section{Resultados}

Durante os três anos que antecederam o censo de serviços que originou este estudo, foram referenciados ou chegaram por demanda espontânea aos Caps de Campinas (SP) 942 indivíduos. Todos eles foram considerados como potenciais participantes da pesquisa AcesSUS. Contudo, apenas 548 usuários (58,2\%) foram encontrados, contatados e convidados a participar do estudo. Os demais $(41,8 \% \mathrm{n}=394)$, inicialmente, foram considerados como perdas da pesquisa.

Entre os 394, por meio dos diários de campo dos coletadores, foi identificado que se tratava de usuários com os quais não foi possível realizar contato telefônico $(18,3 \% \mathrm{n}=72)$; que não tiveram seu prontuário encontrado $(2,3 \%$ $\mathrm{n}=9$ ); que apesar de passar pelo acolhimento, não haviam sido inseridos no serviço (13,4\% $\mathrm{n}=53)$; haviam recebido alta $(37,6 \% \mathrm{n}=148)$; haviam abandonado ou não aderiram ao tratamento ( $12,4 \% \mathrm{n}=49)$; estavam recebendo atendimento domiciliar ou estavam em Serviços Residenciais Terapêuticos $(4,82 \% \mathrm{n}=19)$; haviam sido encaminhados para outros serviços $(2,8 \% \mathrm{n}=11)$; haviam se mudado para outras cidades $(2,5 \% \mathrm{n}=10)$; estavam internados em serviços hospitalares ( $1,5 \% \mathrm{n}=6)$; estavam em situação de rua ( $1,3 \% \mathrm{n}=5)$; estavam privados de liberdade ( $1 \% \mathrm{n}=4)$; estavam desaparecidos $(0,8 \% \mathrm{n}=3)$; ou haviam falecido $(1,3 \% \mathrm{n}=5)$.

Sendo assim, foram considerados, para este estudo, aqueles casos em que, durante a realização do censo de serviços: não foram localizados por contato telefônico; não foram encontrados os prontuários; haviam abandonado ou não aderiram ao tratamento; estavam em situação de rua, ou desaparecidos, totalizando assim 138 usuários. No entanto, durante as visitas aos serviços para investigação desses casos por meio dos prontuários, verificou-se que muitos deles, por serem considerados inativos, estavam no arquivo morto e haviam sido referidos como altas.

Dessa forma, entendeu-se que seria pertinente incluir na investigação deste estudo os 148 casos que, na fase da coleta do censo de serviços, haviam sido reportados nos diários de campo como altas, já que se levantou a suspeita que, entre essas altas, muitas poderiam ser administrativas, encobrindo assim possíveis abandonos ou não adesão ao tratamento. Dessa maneira, na fase de busca em campo, objetivou-se realizar a investigação de 286 prontuários.

Dos 286 prontuários investigados, 60 (21\%) não foram localizados, nem entre os prontuários ativos nem entre aqueles que já haviam sido arquivados. Outros 159 (55,6\%) prontuários estavam referidos como altas; 13 (4,5\%), como abandono ou não adesão ao tratamento; 7 (2,4\%), como casos em que o usuário passou por acolhimento, mas não foi inserido no serviço; 6 (2,1\%) usuários estavam recebendo atenção domiciliar ou estavam em Serviços Residenciais Terapêuticos; 1 (0,3\%) estava privado de liberdade; e outros 40 (14\%) usuários estavam frequentando o serviço de forma regular.

Após investigação dos 159 casos inicialmente reportados como alta nos prontuários, foi possível observar que, na verdade, 57 (35,8\%) casos correspondiam a abandono ou não adesão ao tratamento; 30 (18,9\%), a casos em que o usuário havia se mudado para outras cidades; 40 (25,1\%), a encaminhamentos para atenção básica; 12 (7,5\%), a transferência para outros serviços; 4 (2,5\%), a casos em que o usuário passou por acolhimento, mas não foi inserido no serviço; 4 (2,5\%), a usuários em internação hospitalar; e 2 (1,2\%), a usuários que haviam sido privados de liberdade. Dessa forma, corresponderam a casos efetivos de alta clínica apenas $6,3 \%$ dos casos $(n=10)$.

Por meio da combinação dos resultados obtidos com os diários de campo $(\mathrm{n}=108)$ e dos resultados obtidos na investigação dos prontuários $(n=286)$, foi possível compilar os motivos pelos quais indivíduos que foram referenciados ou chegaram por demanda espontânea aos Caps de Campinas (SP) nos três anos que antecederam o censo de serviços não foram encontrados. O resultado compilado pode ser observado na tabela 1 abaixo. 
Tabela 1. Motivos que levaram a não localização dos potenciais participantes no inquérito de serviços

\begin{tabular}{lrr}
\hline Motivo & $\mathbf{n}$ & $\%$ \\
\hline Usuários que abandonaram ou não aderiam ao tratamento & 70 & $17,8 \%$ \\
Usuários que passaram pelo acolhimento, mas não foram inseridos no serviço & $64,2 \%$ \\
Usuários que não tiveram seu prontuário encontrado & 60 & $15,5 \%$ \\
Usuários referenciados para atenção básica & 40 & $10,2 \%$ \\
Usuários que se mudaram ou estavam fora da cidade & 40 & $10,0 \%$ \\
Usuários que estavam frequentando o serviço, mas não houve sucesso no contato telefônico & 40 & $10,2 \%$ \\
Usuários em atenção domiciliar ou em Serviços Residenciais Terapêuticos & 25 & $6,3 \%$ \\
Usuários que foram transferidos para outros serviços & 23 & $5,8 \%$ \\
Usuários que estavam em internação hospitalar & 10 & $2,5 \%$ \\
Usuários que receberam alta clínica & 10 & $2,5 \%$ \\
Usuários privados de liberdade & 7 & $1,8 \%$ \\
Usuários que foram a óbito & 5 & $1,3 \%$ \\
Total & $\mathbf{3 9 4}$ & $\mathbf{1 0 0} \%$ \\
\hline
\end{tabular}

Fonte: Elaboração própria.

Após a identificação dos usuários que abandonaram ou não aderiram ao tratamento, buscou-se realizar a caracterização deles de acordo com algumas variáveis que estavam disponíveis no prontuário. Os resultados dessa caracterização podem ser observados na tabela 2 a seguir.

Tabela 2. Caracterização dos usuários que abandonaram ou não aderiram ao tratamento

\begin{tabular}{|c|c|c|}
\hline Característica & $\mathrm{n}$ & $\%$ \\
\hline \multicolumn{3}{|l|}{ Sexo } \\
\hline Masculino & 41 & $58,6 \%$ \\
\hline Feminino & 29 & $41,4 \%$ \\
\hline \multicolumn{3}{|l|}{ Idade } \\
\hline 18 a 29 anos & 25 & $37,9 \%$ \\
\hline 30 a 39 anos & 15 & $22,7 \%$ \\
\hline 40 a 49 anos & 11 & $16,7 \%$ \\
\hline 50 a 59 anos & 8 & $12,1 \%$ \\
\hline 60 anos ou mais & 7 & $10,6 \%$ \\
\hline \multicolumn{3}{|l|}{ Estado Civil } \\
\hline Solteiro(a) & 32 & $59,3 \%$ \\
\hline Casado(a) & 17 & $31,5 \%$ \\
\hline Divorciado(a) & 5 & $9,3 \%$ \\
\hline \multicolumn{3}{|l|}{ Cor } \\
\hline Preta & 5 & $15,6 \%$ \\
\hline Parda & 4 & $12,5 \%$ \\
\hline Branca & 23 & $71,9 \%$ \\
\hline
\end{tabular}




\begin{tabular}{lrr}
\hline Tabela 2. (cont.) & $\mathbf{n}$ & $\%$ \\
\hline Característica & \\
\hline Diagnóstico & 33 & $47,1 \%$ \\
\hline Esquizofrenia & 11 & $15,7 \%$ \\
Transtorno afetivo bipolar & 10 & $14,3 \%$ \\
Depressão & 9 & $12,9 \%$ \\
Esquizofrenia concomitante ao uso abusivo de substâncias & 7 & $10 \%$ \\
Outros & 15 & $22,4 \%$ \\
\hline Tempo no Caps & 5 & $7,5 \%$ \\
\hline Até 30 dias & 10 & $14,9 \%$ \\
Até 3 meses & 12 & $17,9 \%$ \\
Até 6 meses & 25 & $37,3 \%$ \\
Até 1 ano & \\
Mais de 1 ano & & \\
\hline
\end{tabular}

Fonte: Elaboração própria.

Conforme observado na tabela 2, a maior parte dos usuários que abandonaram ou não aderiram ao tratamento entre a amostra deste estudo era do sexo masculino $(58,6 \% \mathrm{n}=41)$ e possuíam idade entre 18 e 29 anos $(37,9 \% \mathrm{n}=25)$, sendo a média de idade de 39,9 anos com desvio-padrão de DP=13,7. No que se refere ao estado civil, houve maior proporção de solteiros(as) $(59,3 \% \mathrm{n}=32)$. Quanto à cor, houve uma importante perda de observações (38) devido à ausência de dados no prontuário; contudo, entre os prontuários em que foi possível identificar a cor dos usuários, houve maior proporção de indivíduos de cor branca $(71,9 \% \mathrm{n}=23)$.

Entre os usuários que haviam abandonado ou que não aderiram ao tratamento, o diagnóstico mais prevalente foi esquizofrenia (47,1\% $n=33)$, seguido de transtorno afetivo bipolar (15,7\% $\mathrm{n}=11)$ e depressão $(14,3 \% \mathrm{n}=10)$. Havia ainda nove usuários (12,9\%) que possuíam diagnóstico de esquizofrenia concomitante ao de uso abusivo de substância, e sete (10\%) que apresentavam outros transtornos não especificados. Em relação ao tempo de permanência no Caps, os usuários deste estudo que abandonaram ou não aderiram ao tratamento permaneceram no serviço majoritariamente por mais de um ano $(37,3 \% \mathrm{n}=25)$ ou por menos de um mês $(22,4 \% \mathrm{n}=15)$.

\section{Discussão}

Considerando a literatura existente, é evidente que a condução de estudos com populações oriundas de serviços comunitários de saúde mental no Brasil é suscetível a uma série de dificuldades relacionadas com o rastreio desses indivíduos. Essa perspectiva pode ser observada tanto em estudos com usuários ${ }^{9}$, que apresentaram perdas de 6,6\% de sua amostra, quanto em estudos conduzidos com familiares de usuários"1 em que se observou perdas de até $52,4 \%$ dos potenciais participantes.

Entretanto, cabe ressaltar que, embora sejam apresentados motivos para essas perdas, em geral, não há um estudo aprofundado sobre elas, de forma que se possa caracterizar essa importante parcela de indivíduos que acaba por não ser acessada pelos estudos. Nesse sentido, este trabalho contribui para expansão de uma área ainda pouco explorada dos estudos relativos à da saúde mental comunitária e fornece informações capazes de orientar e potencializar as práticas de pesquisa nesse campo do conhecimento.

Destaca-se que o montante de usuários não encontrados durante a realização do censo de serviços que originou este estudo foi de $41,8 \%$ 
( $\mathrm{n}=394)$ dos indivíduos cadastrados nos Caps III de Campinas nos três anos que antecederam a entrevista. Trata-se de um valor absoluto alto, contudo, difícil de comparar, uma vez que não foram encontrados na literatura estudos com delineamentos de pesquisa semelhantes.

Quanto aos motivos identificados para a impossibilidade de rastreio desses usuários nos serviços durante o período de coleta, pontua-se que $16,2 \%(n=64)$ dos casos estudados eram relativos a pessoas que haviam passado pelo acolhimento nos Caps incluídos neste estudo, porém, não haviam sido, de fato, inseridos no tratamento, provavelmente por não se enquadrarem no perfil de atendimento desses serviços. Cabe destacar que se trata de serviços que trabalham na modalidade porta aberta.

Esse resultado reflete problemas relacionados tanto à forma em que o registro havia sido realizado nos serviços, dando a entender que se tratava de pessoas de interesse para a pesquisa, como problemas em relação ao próprio delineamento do estudo, que não considerou anteriormente quais usuários estavam efetivamente ativos no serviço, sendo, portanto, elegíveis. Dessa forma, evidencia-se a necessidade de as futuras pesquisas contarem com estudos prévios mais detalhados acerca da população que compõe os serviços pesquisados.

Ainda no sentido das limitações oriundas da interface entre a pesquisa e os serviços, deve-se pontuar que $10,2 \%(n=40)$ dos usuários estavam frequentando os serviços, mas não foram localizados por contato telefônico. Esse aspecto está relacionado com a defasagem dos dados cadastrados nos serviços e, também, com a alta rotatividade dos números de contato dos usuários, devido à popularização das linhas de telefonia móvel do tipo pré-pago.

Quanto aos demais motivos que levaram a não localização dos usuários, cabe destacar o alto número de casos que inicialmente foram reportados como altas. Considerando os casos previamente investigados pelos diários de campo e os casos investigados nos serviços, pode-se observar que 159 casos haviam sido reportados pelos serviços como altas. Contudo, após investigação dos prontuários, pode-se observar que se tratava, majoritariamente, de abandonos ou outras situações que levaram à chamada alta administrativa.

Apenas em 2,5\% ( $\mathrm{n}=10)$ dos casos estudados tratava-se de altas clínicas, ou seja, situação em que o usuário não necessitaria mais de acompanhamento no serviço comunitário de saúde mental. Embora o recorte deste estudo não permita uma avaliação profunda sobre o processo de alta nos serviços estudados, por incluir apenas usuários que iniciaram seus tratamentos nos três anos que antecederam a pesquisa, os dados sugerem uma prevalência baixa de altas clínicas. Em um estudo conduzido no município de Joinville (SC) ${ }^{18}$, entre as 621 altas realizadas em um Caps no período de três anos (2014-2016), 46,9\% (n=241) se tratava de altas clínicas. Ressalta-se que, no estudo citado, o Caps em questão mantinha um trabalho específico com as altas no intuito de diminuir o tempo de permanência no Caps e fomentar a inserção no território.

No que diz respeito à assistência em saúde mental no território, cabe destacar que, neste estudo, 10,2\% ( $\mathrm{n}=40)$ dos casos investigados abrangiam pessoas que haviam sido encaminhadas para atenção básica. Essa perspectiva configura um desfecho favorável no cuidado a essa população, uma vez que reflete melhora clínica e possibilidade de transferência do cuidado especializado para outras instâncias, atendendo assim ao preconizado pelas políticas de saúde vigentes no que tange à perspectiva de integração de rede ${ }^{19}$.

Ainda, no que diz respeito à presença de desfechos sugestivamente positivos, cabe pontuar que os resultados deste estudo apontam para a perspectiva de que apenas $2,5 \%(\mathrm{n}=10)$ dos casos estudados estavam passando por uma internação hospitalar à época da pesquisa. Dentro do montante de usuários elegíveis para o estudo ampliado, ou seja, indivíduos inseridos nos serviços nos três anos que antecederam a pesquisa, essa 
proporção seria ainda menor, corroborando os apontamentos de estudos anteriores ${ }^{20}$ de que o Caps seria dispositivo efetivo na redução das internações hospitalares de pessoas com transtornos mentais.

Por outro lado, no que diz respeito a desfechos potencialmente negativos, deve-se considerar que o abandono ou não adesão foi a causa mais frequente para não localização dos indivíduos que compunham a amostra de interesse da pesquisa que originou este estudo.

Os usuários não acessados por abandono ou por não adesão ao tratamento corresponderam a $17,8 \%(n=70)$ dos casos estudados. Trata-se de uma proporção abaixo daquelas encontradas em estudos que investigaram o processo de abandono ou não adesão em pessoas com transtornos mentais. Nesses estudos, as proporções encontradas foram de $22,3 \%{ }^{\mathbf{2 1}} \mathrm{e}$ $39,2 \%{ }^{15}$. Contudo, deve-se considerar as diferenças metodológicas que balizam a comparação dos resultados, já que, neste estudo, a população estudada é composta apenas por indivíduos que foram cadastrados nos serviços nos três anos que antecederam a pesquisa.

Quanto aos demais casos estudados, cabe destacar que não foi possível levantar informações acerca de 15,5\% (n=60) dos indivíduos não acessados pela pesquisa. Trata-se de usuários que tiveram seus prontuários extraviados, e os serviços não souberam informar onde esses registros estavam. Por ser uma proporção significativa dos casos, deve-se considerar que os resultados podem apresentar algum viés em relação à magnitude em que se apresentam, contudo, trazem informações relevantes que possibilitam questionamentos quanto à organização e ao processo de trabalho dos serviços estudados.

No que diz respeito aos usuários que haviam abandonado ou não aderiram ao tratamento, vale ressaltar que houve dificuldades na caracterização desses indivíduos devido à incompletude das informações nos seus registros. Em alguns prontuários, não constavam dados sobre estado civil, cor, ocupação, escolaridade, renda, origem do encaminhamento ou mesmo sobre a composição familiar.
Pontua-se que essa perspectiva tem sido um desafio frequente nas pesquisas conduzidas a partir do uso desses registros. A dificuldade de coleta de dados quanto ao preenchimento deficitário dos prontuários em serviços de saúde mental também foi demonstrada em outros estudos ${ }^{\mathbf{4}, 22,23}$, evidenciando a necessidade de maior sistematização das informações dos usuários.

Com base nas informações disponíveis, foi possível observar que a maioria dos usuários que haviam abandonado ou não aderido ao tratamento nos Caps estudados era do sexo masculino $(58,6 \% \mathrm{n}=41)$. Esse dado vai ao encontro dos resultados pontuados por Pereira et al. ${ }^{17}$ ao investigarem os motivos da evasão de usuários em serviço de saúde mental no município de Lorena (SP).

Também foi observado que, assim como em outros estudos $\mathbf{1 6}^{\mathbf{1 2}, 24,25}$, os indivíduos que haviam abandonado ou não aderido ao tratamento eram majoritariamente de faixas etárias mais jovens. Destaca-se que, em 37,9\% ( $n=$ 25) desses casos, os indivíduos tinham entre 18 e 29 anos, chamando assim atenção para a necessidade de estratégias de vinculação mais efetivas para população jovem.

Quanto à cor dos usuários que haviam abandonado ou não aderido ao tratamento, destaca-se que houve uma proporção maior de indivíduos de cor branca $(71,9 \% \mathrm{n}=23)$. Contudo, cabe ressaltar que esse foi o dado em que mais houve perda de informações relacionadas com a incompletude dos prontuários.

Deve-se levar em conta que as questões relativas à etnicidade e à cor das pessoas são importantes para os estudos referentes ao abandono ou não adesão do tratamento, uma vez que há na literatura registros da contribuição desses fatores para as disparidades vivenciadas por usuários no acesso e na continuidade dos cuidados em serviços de saúde 21,26. A despeito disso, são raros os estudos que trazem análise sobre perfil dos usuários de Caps incluindo essa questão ${ }^{27}$.

Quanto ao diagnóstico, pode-se observar que a maioria dos usuários que haviam 
abandonado ou não aderido ao tratamento possuía diagnóstico de esquizofrenia, correspondendo a $47,1 \%(n=33)$ dos casos. Esse dado, inicialmente, parece contrariar resultados previamente encontrados na literatura, em que há registro de maior prevalência de abandono ou não adesão entre usuários com quadros de transtorno bipolar, depressão e ansiedade $21,24,28$. Contudo, deve-se considerar que este não é um estudo de prevalência, e, sim, caracterização, de modo que os resultados encontrados podem ser influenciados pelas características da população atendida nos serviços estudados.

Deve-se considerar que, no município estudado, a população atendida em serviços especializados é majoritariamente composta por usuários com transtornos graves e persistentes, uma vez que sua rede conta com uma estrutura de atenção básica diferenciada com equipes ampliadas de saúde mental, compostas por psicólogos, psiquiatras e terapeutas ocupacionais. Em decorrência disso, presume-se que os casos mais leves de depressão e ansiedade sejam absorvidos pela atenção básica, e, portanto, não chegam a compor o quadro de usuários dos Caps estudados.

No que diz respeito ao tempo de permanência no serviço, quanto aos usuários que abandonaram ou não aderiram ao tratamento, pode-se observar que a maior parte desses indivíduos havia passado mais de um ano vinculada ao serviço $(37,3 \% \mathrm{n}=25)$. Essa perspectiva contraria os achados por estudos acerca da evasão dos serviços de saúde mental, nos quais houve maior prevalência de abandono em usuários que frequentaram pouco tempo os serviços ${ }^{\mathbf{4} 29}$. Dessa forma, os resultados deste estudo sugerem que as estratégias de adesão dos usuários devem ser pensadas como um processo transversal no tratamento, e não apenas com intuito de vinculação inicial.

Há de se levar em conta que, neste estudo, foi possível caracterizar, com base nas informações disponíveis, quais foram os usuários que haviam abandonado ou não aderido ao tratamento entre os sujeitos que não foram encontrados durante um censo de serviços; contudo, não foi possível identificar quais foram os motivos que levaram ao abandono ou não adesão. Dessa forma, sugere-se que novos estudos sejam realizados no sentido de identificar, por meio da perspectiva dos usuários, quais os fatores que levaram a esse desfecho.

\section{Conclusões}

Os resultados deste estudo apontam para importantes desafios ainda persistentes na condução de pesquisas com os usuários de Caps. Destacam-se aspectos como a desatualização e a incompletude das informações registradas nos prontuários, além da falta de sistematização e extravio dos documentos e de informações acerca dos usuários.

Verificou-se ainda que a alta administrativa se configura como uma prática recorrente nos serviços estudados e ocorre a despeito de situações que deveriam ser mais bem investigadas, como, por exemplo, o abandono e a não adesão do tratamento - motivo mais prevalente para a não localização dos usuários que compuseram os casos apurados neste estudo. Dessa forma, trata-se de uma prática que dificulta o levantamento e a sistematização dos dados acerca da continuidade do tratamento.

Pode-se observar que o grupo de usuários que haviam abandonado ou não aderido ao tratamento era composto, majoritariamente, por homens, jovens, cujo diagnóstico mais frequente era o de esquizofrenia. Quanto ao tempo de permanência, verificou-se que a maioria desses usuários havia passado mais de um ano nos serviços, evidenciando a necessidade de criação de estratégias de adesão como um processo transversal durante todo o tratamento, e não somente no momento da vinculação inicial.

Nesse sentido, apesar deste não se tratar de um estudo de prevalência e investigação dos fatores associados ao abandono e não adesão ao 
tratamento, os seus resultados trazem informações relevantes que possibilitam importantes reflexões quanto à organização e ao processo de trabalho dos serviços estudados e, ainda, para a melhoria do seguimento dos usuários.

\section{Agradecimentos}

À Fundação de Amparo à Pesquisa do Estado de Mato Grosso (Fapemat), pelo apoio financeiro mediante concessão da bolsa de doutorado. Ao Conselho Nacional de Desenvolvimento Científico e Tecnológico (CNPq) pelo financiamento da Pesquisa AcesSUS.

\section{Referências} and Contributor ID).

\section{Colaboradores}

Bandeira N (0000-0003-4391-5414)* contribuiu para a concepção, o planejamento, a análise e interpretação dos dados, elaboração do rascunho, revisão critica do conteúdo e a aprovação da versão final do manuscrito. Treichel CAS (0000-0002-0440-9108)* contribuiu para a análise e interpretação de dados e redação do manuscrito. Onocko-Campos RT (0000-0003-0469-5447)* contribuiu para a concepção e delineamento da pesquisa, análise e interpretação dos dados e revisão crítica do manuscrito.
1. Brasil. Lei $n^{0} 10.216$, de 06 de abril de 2001. Dispõe sobre a proteção e os direitos das pessoas portadoras de transtornos mentais e redireciona o modelo assistencial em saúde mental. [internet]. Diário Oficial da União. 6 Abr 2001. [acesso em 2018 maio 20]. Disponível em: http://www.saude.mg.gov.br/images/ documentos/lei_10216.pdf.

2. Brasil. Ministério da Saúde. Portaria n.o 336, de 19 de fevereiro de 2002. Regulamenta a constituição e funcionamento dos Centros de Atenção Psicossocial [internet]. Diário Oficial da União. 20 Fev 2002. [acesso em 2018 maio 20]. Disponível em: http://www.saude. mg.gov.br/images/documentos/Portaria_336.pdf.

[internet]. 12. ed. Brasília, DF: MS; 2015 [acesso em 2018 maio 20]. Disponível em: https://www.mhinnovation.net/sites/default/files/downloads/innovation/reports/Report_12-edicao-do-Saude-Mental-em-Dados.pdf.

4. Pereira MO, Souza JDM, Maria Â. Perfil dos usuários de serviços de Saúde Mental do município de Lorena - São Paulo. Acta paul. enferm. 2012; 25(11):48-54.

5. Kantorski LP, Jardim VMDR, Wetzel C, et al. Satisfação dos usuários dos centros de atenção psicossocial da região Sul do Brasil. Rev Saúde Pública. 2009; 43(supl1):29-35.

3. Brasil. Ministério da Saúde. Saúde Mental em Dados 
ças percebidas em usuários de Centros de Atenção Psicossocial do Sul do Brasil a partir de sua inserção nos serviços. Saúde debate. 2018; 42(esp4):166-74.

7. Treichel CAS, Jardim VMR, Aldrigui LB. Conduzindo Pesquisa com familiares de usuários de Centros de Atenção Psicossocial: Planejamento de Campo e controle de perdas. In: Anais do I Congresso Internacional de Pesquisa em Enfermagem e Saúde [internet]; 2017 Set 21-22; Pelotas. Pelotas: Universidade de Pelotas; 2017 [acesso em 2019 set 10]. Disponível em: https://wp.ufpel.edu.br/cipes/files/2017/11/Anais-do-I-CIPES-4.pdf.

8. Cavalcanti MT, Dahl CM, Valencia E, et al. Critérios de admissão e continuidade de cuidados em centros de atenção psicossocial, Rio de Janeiro, RJ. Rev Saude Publica [internet]. 2009 [acesso em 2018 maio 20]; 43(supl.1):23-8. Disponível em: https://scielosp.org/ article/rsp/2009.v43suppll/23-28/en/.

9. Silva SN, Lima MG, Ruas CM. Avaliação de Serviços de Saúde Mental Brasileiros : satisfação dos usuários e fatores associados Brazilian Mental Health Services Assessment: user satisfaction and associated factors. Ciênc. Saúde Colet. 2018; 23(11):3799-810.

10. Treichel CAS, Jardim VMR, Kantorski LP, et al. Prevalence and factors associated with suicidal ideation among family caregivers of people with mental disorders. J Clin Nurs. 2019; 28(19-20):1-8.

11. Tabeleão VP, Tomasi E, Quevedo LÁ. Sobrecarga de familiares de pessoas com transtorno psíquico : níveis e fatores associados. Rev Psiq Clin. 2014;41(3):63-6.

12. Oliveira GL, Waleska Teixeira C, Cherchiglia ML. Saúde mental e a continuidade do cuidado em centros de saúde de Belo Horizonte, MG. Rev Saude Publica [internet]. 2008 [acesso em 2018 maio 20]; 42(4):707-16. Disponível em: http://www.scielo.br/scielo.php?script=sci_arttext $\&$ pid=S0034$-89102008000400018 \& \operatorname{lng}=\mathrm{en}$.

13. Basu A, Arya S. Drop out from treatment in the World Mental Health Survey initiative. Br J Psychiatry [internet]. 2013 [acesso em 2018 maio 20]; 202(05):383.
Disponível em: Https://www.cambridge.org/core/ product/identifier/S0007125000274503/type/journal_article.

14. Wells JE, Browne MO, Aguilar-Gaxiola S, et al. Drop out from out-patient mental healthcare in the World Health Organization's World Mental Health Survey initiative [internet]. 2013 [acesso em 2018 maio 20]; Diponível em: http://www.thehealthwell.info/node /693959?\&content=resource\&member $=6841 \&$ catalo gue=none $\&$ collection=none $\&$ tokens_complete=true.

15. Melo APS, Guimarães MDC. Factors associated with psychiatric treatment dropout in a mental health reference center, Belo Horizonte. Rev Bras Psiquiatr [internet]. 2005 [acesso em 2018 maio 15]; 27(2):113-8. Disponível em: http://www.scielo.br/scielo.php?script=sci_arttext\&pid=S1516$-44462005000200008 \& \operatorname{lng}=$ en $\&$ tlng=en.

16. Ribeiro MS, Alves MJM, Vieira EMM, et al. Fatores associados ao abandono de tratamento em saúde mental em uma unidade de nível secundário do Sistema Municipal de Saúde TT - Factors associated to treatment dropout in mental health. J Bras Psiquiatr [internet]. 2008 [acesso em 2018 maio 20]; 57(1):16-22. Disponível em: http://www.scielo.br/scielo.php?script=sci arttext\&pid=S0047-20852008000100004.

17. Pereira MO, Amorim A, Vidal V, et al. Busca ativa para conhecer o motivo da evasão de usuários em serviço de saúde mental. ACTA Paul Enferm. 2013; 26(5):409-12.

18. Kantorski LP, Guedes AC, Machado RA, et al. A alta no centro de atenção psicossocial. J Nurs Heal. 2019; 9(3):1-10.

19. Brasil. Ministério da Saúde. Portaria no 4.279 , de 30 de dezembro de 2010 [internet]. Diário Oficial da União. 31 Dez 2010 [acesso em 2018 maio 20]. Disponível em: http://bvsms.saude.gov.br/bvs/saudelegis/gm/2010/prt4279_30_12_2010.html.

20. Tomasi E, Facchini LA, Piccini RX, et al. Efetividade dos Centros de Atenção Psicossocial no cuidado a portadores de sofrimento psíquico em cidade de 
porte médio do Sul do Brasil: Uma análise estratificada. Cad. Saúde Pública. 2010; 26(4):807-15.

21. Wang J. Mental Health Treatment Dropout and Its correlates in a General Pupulation Sample. Med Care. 2007;45(3):224-9.

22. Pelisoli CL, Moreira ÂK. Caracterização epidemiológica dos usuários do Centro de Atenção Psicossocial Casa Aberta. Rev Psiquiatr RS. 2005; 27(3):270-7.

23. Bellettini F, Gomes KM. Perfil dos usuários do centro de atenção psicossocial e do programa de saúde mental no município de Orleans-SC. Cad Bras Saúde Ment. 2013; 5(12):161-75.

24. Henzen A, Moeglin C, Giannakopoulos P, et al. Determinants of dropout in a community-based mental health crisis centre. BMC Psychiatry [internet]. 2016 [acesso em 2018 maio 20]; 16(1):111. Disponível em: http://bmcpsychiatry.biomedcentral.com/articles/10.1186/s12888-016-0819-4.

25. Marcus MA, Westra HA, Eastwood JD, et al. What are young adults saying about mental health? An analysis of Internet blogs. J Med Internet Res [internet]. 2012 [acesso em 2018 maio 20]; 14(1):el7. Disponível em: http://www.jmir.org/2012/1/e17/.
26. Jimenez DE, Cook B, Bartels SJ, et al. Disparities in mental health service use of racial and ethnic minority elderly adults. J Am Geriatr Soc [internet]. 2013 [acesso em 2018 maio 20]; 61(1):18-25. Diponível em: http://doi.wiley.com/10.1111/jgs.12063.

27. Silva NG. The race/color variable in studies of characterization of the users of Psychosocial Care Centers. 2017; 26(1):100-14.

28. Lora A. An overview of the mental health system in Italy. Ann Ist Super Sanita [internet]. 2009 [acesso em 2018 maio 20]; 45(1):5-16. Disponível em: http:// www.ncbi.nlm.nih.gov/pubmed/19567972.

29. Simon GE, Imel ZE, Ludman EJ, et al. Is dropout after a first psychotherapy visit always a bad outcome? Psychiatr Serv [internet]. 2012 [acesso em 2018 maio 20]; 63(7):705-7. Disponível em: http://psychiatryonline.org/doi/abs/10.1176/appi.ps.201100309.

Recebido em 16/09/2019

Aprovado em 14/05/2020

Conflito de interesses: inexistente

Suporte financeiro: Fundação de Amparo à Pesquisa do Estado de Mato Grosso (Fapemat), édital no 09/2016 e Conselho Nacional de Desenvolvimento Científico e Tecnológico (CNPq), Chamada MCTI/CNPq/CT-Saúde/MS/SCTIE/Decit N ㅇ 41/2013. 